Mathematical Modelling and Analysis

Volume 16 Number 1, March 2011, 82-96

Doi:10.3846/13926292.2011.560617

(C) Vilnius Gediminas Technical University, 2011
www.tandf.co.uk/journals/TMMA

Publisher: Taylor\&Francis and VGTU

Online ISSN: 1648-3510

Print ISSN: 1392-6292

\title{
Nordsieck Methods with Inherent Quadratic Stability*
}

\section{Braś}

Department of Applied Mathematics, AGH University of Science and Technology al. Mickiewicza 30, 30059 Kraków, Poland E-mail: brasmich@wms.mat.agh.edu.pl

Received August 24, 2010; revised January 6, 2011; published online March 1, 2011

Abstract. We derive sufficient conditions which guarantee that the stability polynomial of Nordsieck method for ordinary differential equations has only two nonzero roots. Examples of such methods up to order four are presented which are $A$ - and $L$-stable. These examples were obtained by computer search using the Schur criterion applied to the quadratic factor of the resulting stability polynomials.

Keywords: Nordsieck methods, general linear methods, order conditions, inherent quadratic stability.

AMS Subject Classification: 65L05; 65L20.

\section{Introduction}

We consider the class of Nordsieck methods for ordinary differential equations (ODEs)

$$
\left\{\begin{array}{l}
y^{\prime}(t)=f(y(t)), \quad t \in\left[t_{0}, T\right], \\
y\left(t_{0}\right)=y_{0} \in \mathbb{R}^{m},
\end{array}\right.
$$

where $f: \mathbb{R}^{m} \rightarrow \mathbb{R}^{m}$ is sufficiently smooth. These methods are defined by

$$
\begin{cases}Y_{i}^{[n]}=h \sum_{j=1}^{s} a_{i j} f\left(Y_{j}^{[n]}\right)+\sum_{j=1}^{r} u_{i j} z_{j}^{[n-1]}, & i=1,2, \ldots, s, \\ z_{i}^{[n]}=h \sum_{j=1}^{s} b_{i j} f\left(Y_{j}^{[n]}\right)+\sum_{j=1}^{r} v_{i j} z_{j}^{[n-1]}, & i=1,2, \ldots, r .\end{cases}
$$

Here $Y_{i}^{[n]}$ are approximations of stage order $q$ to $y\left(t_{n}+c_{i} h\right), i=1,2, \ldots, s$, and $z_{i}^{[n]}$ are approximations of order $p$ to the scaled derivatives $h^{i-1} y^{(i-1)}\left(t_{n}\right)$,

* This research was partially supported by the Polish Ministry of Science and Higher Education. 
$i=1,2, \ldots, r$. These methods form a subclass of general linear methods (GLM) $[3,4,18]$ for ODEs. They are represented by the vector $\mathbf{c}=\left[c_{1}, \ldots, c_{s}\right]^{T}$, and four coefficient matrices $\mathbf{A} \in \mathbb{R}^{s \times s}, \mathbf{U} \in \mathbb{R}^{s \times r}, \mathbf{B} \in \mathbb{R}^{r \times s}, \mathbf{V} \in \mathbb{R}^{r \times r}$.

In this paper we assume that $c_{i} \neq c_{j}$ and that $r=s+1$, where $r$ is the number of external approximations and $s$ is the number of stages or internal approximations. We are interested in methods of order $p=s$ and stage order $q=p$. Order and stage order conditions will be discussed in Section 2 .

To facilitate efficient implementation we will always assume that the coefficient matrix $\mathbf{A}$ has the form

$$
\mathbf{A}=\left[\begin{array}{ccccc}
\lambda & & & & \\
a_{21} & \lambda & & & \\
\vdots & \vdots & \ddots & & \\
a_{s-1,1} & a_{s-1,2} & \cdots & \lambda & \\
a_{s, 1} & a_{s, 2} & \cdots & a_{s, s-1} & \lambda
\end{array}\right]
$$

To ensure that the method (1.2) is zero-stable we will also assume that the coefficient matrix $\mathbf{V}$ has the form

$$
\mathbf{V}=\left[\begin{array}{ccccc}
1 & v_{12} & v_{13} & \cdots & v_{1, s+1} \\
0 & 0 & v_{23} & \cdots & v_{2, s+1} \\
\vdots & \vdots & \vdots & \ddots & \vdots \\
0 & 0 & 0 & \cdots & v_{s, s+1} \\
0 & 0 & 0 & \cdots & 0
\end{array}\right]
$$

Applying (1.2) to the linear test equation

$$
y^{\prime}=\xi y, \quad t \geq 0
$$

$\xi \in \mathbb{C}$, we obtain the recurrence equation

$$
z^{[n]}=\mathbf{M}(z) z^{[n-1]}, \quad n=0,1, \ldots,
$$

$z=h \xi$, with the stability matrix $\mathbf{M}(z)$ defined by

$$
\mathbf{M}(z)=\mathbf{V}+z \mathbf{B}(\mathbf{I}-z \mathbf{A})^{-1} \mathbf{U}
$$

We also define the stability function of the method (1.2) as the characteristic polynomial of $\mathbf{M}(z)$, i.e.,

$$
p(w, z)=\operatorname{det}(w \mathbf{I}-\mathbf{M}(z)) .
$$

This function is a polynomial of degree $s+1$ with respect to $w$ and coefficients are rational functions with respect to $z$. To investigate stability properties of methods corresponding to this function it is usually more convenient to work with the polynomial obtained by multiplying (1.3) by its denominator. Then 
the coefficients of the resulting polynomial with respect to $w$, which will be denoted by the same symbol $p(w, z)$, are polynomials with respect to $z$.

Nordsieck form of general linear methods are often used in variable stepsize and variable order implementations and for error estimations (see [9]). Nordsieck form of two-step Runge-Kutta methods [20] was introduced by Bartoszewski and Jackiewicz [1]. Nordsieck representation of DIMSIMs was studied in [5]. Some implementation details and results of experiments can be found in $[6,7,17]$. DIMSIMs methods make a subclass of Nordsieck methods investigated in this paper (see, also [8]). Nordsieck methods are useful to solve differential systems resulting from semi discretization of PDE modelling various phenomena in science and engineering. Examples of such systems can be found in $[19,11]$.

Recent research is also concerned with algebraic stability of Nordsieck methods $[2,15]$. Criteria for algebraic stability of general linear methods given by Hill [16] was used to construct algebraically stable Nordsieck methods up to order 4. For the two-step Runge-Kutta methods with algebraic stability see [13].

The organization of this paper is as follows. In Section 2 we derive order and stage order conditions and representation formulas for coefficient matrices of the method (1.2). In Section 3 we present some conditions which guarantee that stability function has only two nonzero roots. These results are based on work of D'Ambrosio, Izzo and Jackiewicz [12] for two-step Runge-Kutta methods. In Sections 4, 5 and 6 we use these criteria to construct methods up to order 4. Results of numerical experiments are given in Section 7. In Section 8 some concluding remarks are presented.

\section{Order and Stage Order Conditions}

In this section we present order and stage order conditions for Nordsieck methods. Define the vector $Z=\left[1, z, \ldots, z^{p}\right]^{T}, z \in \mathbb{C}$ and assume that $r=s+1$. Then it follows from the theory of order conditions for GLM (see, for example, $[3,4,18,22])$ that the method $(1.2)$ has order $p=s$, i.e.,

$$
z_{i}^{[n-1]}=h^{i-1} y^{(i-1)}\left(t_{n-1}\right)+O\left(h^{s+1}\right)
$$

implies

$$
z_{i}^{[n]}=h^{i-1} y^{(i-1)}\left(t_{n}\right)+O\left(h^{s+1}\right),
$$

$i=1,2, \ldots, r$, and stage order $q=p$, i.e.,

$$
Y_{i}^{[n]}=y\left(t_{n-1}+c_{i} h\right)+O\left(h^{s+1}\right), \quad i=1,2, \ldots, s,
$$

if and only if

$$
\begin{aligned}
& e^{\mathbf{c} z}=z \mathbf{A} e^{\mathbf{c} z}+\mathbf{U} Z+O\left(z^{p+1}\right), \\
& e^{z} Z=z \mathbf{B} e^{\mathbf{c} z}+\mathbf{V} Z+O\left(z^{p+1}\right),
\end{aligned}
$$


where $e^{\mathbf{c} z}=\left[e^{c_{1} z}, e^{c_{2} z}, \ldots, e^{c_{s} z}\right]^{T}$. These conditions can be used to express the coefficient matrices $\mathbf{U}$ and $\mathbf{B}$ in terms of $\mathbf{A}, \mathbf{V}$ and $\mathbf{c}$. Define the matrices $\mathbf{K}_{p} \in \mathbb{R}^{(p+1) \times(p+1)}$ and $\mathbf{E}_{p} \in \mathbb{R}^{(p+1) \times(p+1)}$ by

$$
\mathbf{K}_{p}=\left[\begin{array}{ccccc}
0 & 1 & 0 & \cdots & 0 \\
0 & 0 & 1 & \cdots & 0 \\
\vdots & \vdots & \vdots & \ddots & \vdots \\
0 & 0 & 0 & \cdots & 1 \\
0 & 0 & 0 & \cdots & 0
\end{array}\right], \quad \mathbf{E}_{p}=\exp \left(\mathbf{K}_{p}\right)=\left[\begin{array}{ccccc}
1 & 1 & \frac{1}{2 !} & \cdots & \frac{1}{p !} \\
0 & 1 & 1 & \cdots & \frac{1}{(p-1) !} \\
0 & 0 & 1 & \cdots & \frac{1}{(p-2) !} \\
\vdots & \vdots & \vdots & \ddots & \vdots \\
0 & 0 & 0 & \cdots & 1
\end{array}\right],
$$

and the matrix $\mathbf{C}_{p}=\left[\mathbf{e}, \mathbf{c}, \frac{\mathbf{c}^{2}}{2 !}, \cdots, \frac{\mathbf{c}^{p}}{p !}\right] \in \mathbb{R}^{p \times(p+1)}$, where $\mathbf{c}^{i}$ stands for componentwise exponentiation. We have the following result.

Theorem 1 [see $[10,18,22]]$. Assume that $p=q=s$ and $r=s+1$. Then

$$
\begin{aligned}
\mathbf{U} & =\mathbf{C}_{p}-\mathbf{A C}_{p} \mathbf{K}_{p}, \\
\mathbf{V} & =\mathbf{E}_{p}-\mathbf{B C}_{p} \mathbf{K}_{p} .
\end{aligned}
$$

Proof. Expanding $e^{\mathbf{c} z}$ into Taylor series we obtain that:

$$
e^{\mathbf{c} z}=\left[\begin{array}{c}
e^{c_{1} z} \\
\vdots \\
e^{c_{p} z}
\end{array}\right]=\left[\begin{array}{c}
\sum_{j=0}^{p} \frac{c_{1}^{j} z^{j}}{j !} \\
\vdots \\
\sum_{j=0}^{p} \frac{c_{p}^{j} z^{j}}{j !}
\end{array}\right]+O\left(z^{p+1}\right)=\mathbf{C}_{p} Z+O\left(z^{p+1}\right) .
$$

Since $z \cdot Z=\mathbf{K}_{p} Z+O\left(z^{p+1}\right)$, it follows from (2.5) that

$$
z e^{\mathbf{c} z}=\mathbf{C}_{p} \mathbf{K}_{p} Z+O\left(z^{p+1}\right) .
$$

Substituting (2.5) and (2.6) into (2.1) we obtain relation that is equivalent to (2.3). Similarly, taking into account that $e^{z} Z=\mathbf{E}_{p} Z+O\left(z^{p+1}\right)$ from condition (2.2) we obtain (2.4). This completes the proof.

We are interested in expressing the coefficient matrix $\mathbf{B}$ in terms of $\mathbf{c}$ and $\mathbf{V}$ instead of (2.4). We partition the matrices $\mathbf{B}, \mathbf{V}, \mathbf{E}_{p}$ and $\mathbf{C}_{p}$ as follows

$$
\mathbf{B}=\left[\begin{array}{c}
\mathbf{b}^{T} \\
\hline \widetilde{\mathbf{B}}
\end{array}\right], \mathbf{V}=\left[\begin{array}{c|c}
\mathbf{1} & \mathbf{v} \\
\hline \mathbf{0} & \widetilde{\mathbf{V}}
\end{array}\right], \mathbf{E}_{p}=\left[\begin{array}{c|c}
\mathbf{1} & \mathbf{e}_{p-1}^{T} \\
\hline \mathbf{0} & \mathbf{E}_{p-1}
\end{array}\right], \mathbf{C}_{p}=\left[\mathbf{C}_{p-1} \mid \frac{\mathbf{c}^{p}}{p !}\right],
$$

where $\mathbf{b}^{T}$ stands for first row of $\mathbf{B}$ and $\mathbf{0}$ stands for vector or matrix of appropriate dimension. We have the following theorem.

Theorem 2 [see [18]]. Assume that $p=q=s, r=s+1$ and that the components of abscissa vector $\mathbf{c}$ are distinct. Then

$$
\begin{aligned}
& \mathbf{b}^{T}=\left(\mathbf{e}_{p-1}^{T}-\mathbf{v}\right) \mathbf{C}_{p-1}^{-1}, \\
& \widetilde{\mathbf{B}}=\left(\mathbf{E}_{p-1}-\tilde{\mathbf{V}}\right) \mathbf{C}_{p-1}^{-1} .
\end{aligned}
$$


Proof. From (2.4) and the relation $\mathbf{C}_{p} \mathbf{K}_{p}=\left[\mathbf{0} \mid \mathbf{C}_{p-1}\right]$ it follows that

$$
\left[\begin{array}{c|c}
\mathbf{1} & \mathbf{v} \\
\hline \mathbf{0} & \widetilde{\mathbf{V}}
\end{array}\right]=\left[\begin{array}{c|c}
\mathbf{1} & \mathbf{e}_{p-1}^{T} \\
\hline \mathbf{0} & \mathbf{E}_{p-1}
\end{array}\right]-\left[\begin{array}{c|c}
0 & \mathbf{b}^{T} \mathbf{C}_{p-1} \\
\hline \mathbf{0} & \widetilde{\mathbf{B}} \mathbf{C}_{p-1}
\end{array}\right] .
$$

Comparing the corresponding elements on both sides of this matrix equation and from assumptions of the theorem that the matrix $\mathbf{C}_{p-1}$ is invertible we obtain (2.7) and (2.8).

\section{Criteria for Quadratic Stability}

The stability function (1.3) multiplied by its denominator is the polynomial of the form

$$
p(w, z)=(1-\lambda z)^{s} w^{s+1}-p_{s}(z) w^{s}+\cdots+(-1)^{s} p_{1}(z) w+(-1)^{s+1} p_{0}(z),
$$

where

$$
\begin{aligned}
p_{s}(z) & =1+p_{s 1} z+\cdots+p_{s s} z^{s} \\
p_{s-1}(z) & =p_{s-1,1} z+\cdots+p_{s-1, s} z^{s} \\
& \vdots \\
p_{0}(z) & =p_{01} z+\cdots+p_{0 s} z^{s} .
\end{aligned}
$$

In the case $p=q=s$ the coefficients $p_{i j}$ of the polynomials $p_{i}(z)$ depend on $\mathbf{A}, \mathbf{V}$ and $\mathbf{c}$.

A direct idea to obtain methods whose stability function has only two nonzero roots is to compute stability function (3.1) and to solve system of nonlinear equations $p_{i j}=0, i=0, \ldots, s-2, j=1, \ldots, s$. Formulating and solving this system is a very difficult task, so it is interesting to search for some other conditions which will guarantee this property.

Results in this section are based on those obtained by Conte, D'Ambrosio and Jackiewicz [12]. To investigate the Nordsieck methods with so called quadratic stability function, i.e stability function with two nonzero roots, we introduce equivalence relation between matrices of the same dimensions. We say that two matrices $\mathbf{D}$ and $\mathbf{E}$ are equivalent, denoted by $\mathbf{D} \equiv \mathbf{E}$, if they are equal except for the first two rows $[12,18]$.

For matrix $\mathbf{F} \in \mathbb{R}^{(s+1) \times n}, n \in \mathbb{N}$ we have that $\mathbf{D} \equiv \mathbf{E} \Rightarrow \mathbf{D F} \equiv \mathbf{E F}$.

Definition 1 [see $[12,18]]$. The Nordsieck method with coefficients matrices $\mathbf{A}, \mathbf{U}, \mathbf{B}, \mathbf{V}$ has inherent quadratic stability (IQS) if there exists a matrix $\mathbf{X} \in \mathbb{R}^{(s+1) \times(s+1)}$ such that

$$
\begin{aligned}
& \mathbf{B A} \equiv \mathbf{X B} \\
& \mathbf{B U} \equiv \mathbf{X V}-\mathbf{V X} .
\end{aligned}
$$

Similarly as in [18] and [12] we have the following result. 
Theorem 3. Assume that the Nordsieck method has IQS. Then its stability function $p(w, z)$ assumes the form

$$
p(w, z)=w^{s-1}\left(w^{2}-p_{1}(z) w+p_{0}(z)\right),
$$

where $p_{1}(z)$ and $p_{0}(z)$ are rational functions with respect to $z$.

Proof. The proof is similar to that in [18] and [12]. We observe that (3.2) is equivalent to

$$
\mathbf{B}(\mathbf{I}-z \mathbf{A}) \equiv(\mathbf{I}-z \mathbf{X}) \mathbf{B} .
$$

Assuming that $\mathbf{I}-z \mathbf{A}$ in nonsingular it follows that

$$
\mathbf{B} \equiv(\mathbf{I}-z \mathbf{X}) \mathbf{B}(\mathbf{I}-z \mathbf{A})^{-1} .
$$

Instead of stability matrix $\mathbf{M}(z)$ we consider similar matrix $\widetilde{\mathbf{M}}(z)$ defined below. Using (3.3) and (3.5) and assuming that $\mathbf{I}-z \mathbf{X}$ is nonsingular it follows that

$$
\begin{aligned}
\widetilde{\mathbf{M}}(z) & =(\mathbf{I}-z \mathbf{X}) \mathbf{M}(z)(\mathbf{I}-z \mathbf{X})^{-1} \\
& =(\mathbf{I}-z \mathbf{X})\left(\mathbf{V}+z \mathbf{B}(\mathbf{I}-z \mathbf{A})^{-1} \mathbf{U}\right)(\mathbf{I}-z \mathbf{X})^{-1} \\
& =\left(\mathbf{V}-z \mathbf{X} \mathbf{V}+z(\mathbf{I}-z \mathbf{X}) \mathbf{B}(\mathbf{I}-z \mathbf{A})^{-1} \mathbf{U}\right)(\mathbf{I}-z \mathbf{X})^{-1} \\
& \equiv(\mathbf{V}-z \mathbf{X} \mathbf{V}+z \mathbf{B U})(\mathbf{I}-z \mathbf{X})^{-1} \\
& \equiv(\mathbf{V}-z \mathbf{X} \mathbf{V}+z(\mathbf{X} \mathbf{V}-\mathbf{V X}))(\mathbf{I}-z \mathbf{X})^{-1} \\
& =(\mathbf{V}-z \mathbf{V X})(\mathbf{I}-z \mathbf{X})^{-1}
\end{aligned}
$$

Hence, $(\mathbf{I}-z \mathbf{X}) \mathbf{M}(z)(\mathbf{I}-z \mathbf{X})^{-1} \equiv \mathbf{V}$. We can write this relation in the following form

$$
(\mathbf{I}-z \mathbf{X}) \mathbf{M}(z)(\mathbf{I}-z \mathbf{X})^{-1}=\left[\begin{array}{cc|cccc}
\widetilde{\mathbf{M}}_{11}(z) & \multicolumn{5}{|c}{\widetilde{\mathbf{M}}_{12}(z)} \\
\hline 0 & 0 & 0 & v_{34} & \ldots & v_{3, s+1} \\
\vdots & \vdots & \vdots & \vdots & \ddots & \vdots \\
0 & 0 & 0 & 0 & \ldots & v_{s, s+1} \\
0 & 0 & 0 & 0 & \ldots & 0
\end{array}\right]
$$

where $\widetilde{\mathbf{M}}_{11}(z) \in \mathbb{R}^{2 \times 2}$, and $\widetilde{\mathbf{M}}_{12}(z) \in \mathbb{R}^{2 \times(s-1)}$. Hence, the characteristic function $p(w, z)$ of $\widetilde{\mathbf{M}}(z)$ and $\mathbf{M}(z)$ assumes the form (3.4). This completes the proof.

\section{Construction of Methods with $s=1$ and $s=2$}

Use of representation formulas $(2.3),(2.7)$ and (2.8) for $p=q=s=1$ leads to a three-parameter family of methods depending on $c_{1}, \lambda$, and $v_{12}$. The coefficients of these methods are

$$
\left[\begin{array}{c|c}
\mathbf{A} & \mathbf{U} \\
\hline \mathbf{B} & \mathbf{V}
\end{array}\right]=\left[\begin{array}{c|cc}
\lambda & 1 & c_{1}-\lambda \\
\hline 1-v_{12} & 1 & v_{12} \\
1 & 0 & 0
\end{array}\right]
$$


and the stability polynomial takes the form

$$
p(w, z)=(1-\lambda z) w^{2}-p_{1}(z) w+p_{0}(z),
$$

where

$$
p_{1}(z)=1+\left(1+c_{1}-2 \lambda-v_{12}\right) z, \quad p_{0}(z)=\left(c_{1}-\lambda-v_{12}\right) z .
$$

This polynomial is already of degree 2 with respect to $w$. Taking $c_{1}=1$, $\lambda=1, v_{12}=0$ we obtain the backward Euler formula given by

$$
\left[\begin{array}{c|c}
\mathbf{A} & \mathbf{U} \\
\hline \mathbf{B} & \mathbf{V}
\end{array}\right]=\left[\begin{array}{l|ll}
1 & 1 & 0 \\
\hline 1 & 1 & 0 \\
1 & 0 & 0
\end{array}\right]
$$

This method is $A$-stable and algebraically stable.

Case $p=q=s=2$ is nontrivial. First we use (2.3), (2.7), and (2.8) and obtain that the matrices $\mathbf{B}$ and $\mathbf{U}$ are given by

$$
\begin{aligned}
& \mathbf{U}=\left[\begin{array}{ccc}
1 & c_{1}-\lambda & \left(c_{1}^{2}-2 \lambda c_{1}\right) / 2 \\
1 & c_{2}-\lambda-a_{21} & \left(c_{2}^{2}-2 \lambda c_{2}-2 a_{21} c_{1}\right) / 2
\end{array}\right], \\
& \mathbf{B}=\left[\begin{array}{cc}
\frac{1-2 c_{2}+2 c_{2} v_{12}-2 v_{13}}{2\left(c_{1}-c_{2}\right)} & \frac{-1+2 c_{1}-2 c_{1} v_{12}+2 v_{13}}{2\left(c_{1}-c_{2}\right)} \\
\frac{1-c_{2}-v_{23}}{c_{1}-c_{2}} & \frac{-1+c_{1}+v_{23}}{c_{1}-c_{2}} \\
1 /\left(c_{1}-c_{2}\right) & -1 /\left(c_{1}-c_{2}\right)
\end{array}\right] .
\end{aligned}
$$

Solving conditions (3.2) and (3.3) with respect to coefficients of matrix $\mathbf{X}, a_{21}$ and $v_{23}$ we obtain

$$
\begin{aligned}
& \mathbf{A}=\left[\begin{array}{cc}
\lambda & 0 \\
c_{2}-c_{1} & \lambda
\end{array}\right], \quad \mathbf{U}=\left[\begin{array}{ccc}
1 & c_{1}-\lambda & \frac{1}{2}\left(c_{1}^{2}-2 c_{1} \lambda\right) \\
1 & c_{1}-\lambda & \frac{1}{2}\left(2 c_{1}^{2}-2 c_{2} c_{1}+c_{2}^{2}-2 c_{2} \lambda\right)
\end{array}\right], \\
& \mathbf{B}=\left[\begin{array}{cc}
\frac{2 v_{12} c_{2}-2 c_{2}-2 v_{13}+1}{2\left(c_{1}-c_{2}\right)} & \frac{-2 v_{12} c_{1}+2 c_{1}+2 v_{13}-1}{2\left(c_{1}-c_{2}\right)} \\
\frac{c_{1}-3 c_{2}+2 \lambda+2}{2\left(c_{1}-c_{2}\right)} & \frac{c_{1}+c_{2}-2 \lambda-2}{2\left(c_{1}-c_{2}\right)} \\
1 /\left(c_{1}-c_{2}\right) & 1 /\left(c_{2}-c_{1}\right)
\end{array}\right], \\
& \mathbf{V}=\left[\begin{array}{ccc}
1 & v_{12} & v_{13} \\
0 & 0 & \frac{1}{2}\left(-c_{1}+c_{2}-2 \lambda\right) \\
0 & 0 & 0
\end{array}\right] .
\end{aligned}
$$

The stability polynomial of such method for $\mathbf{c}=[0,1]^{T}$ is

$$
p(w, z)=w^{3}(\lambda z-1)^{2}-w^{2} p_{1}(z)+w p_{0}(z),
$$


where

$$
\begin{aligned}
& p_{1}(z)=\frac{1}{2}\left(4 \lambda^{2} z^{2}+2 \lambda v_{12} z^{2}-4 \lambda z^{2}-8 \lambda z-2 v_{12} z-2 v_{13} z^{2}+z^{2}+3 z+2\right), \\
& p_{0}(z)=\frac{1}{2}\left(2 \lambda^{2} z^{2}+2 \lambda v_{12} z^{2}-4 \lambda z^{2}-4 \lambda z-2 v_{12} z^{2}-2 v_{12} z-2 v_{13} z^{2}+z^{2}+z\right) .
\end{aligned}
$$

The method is $L$-stable if $p_{22}=0$ and $p_{12}=0$ (compare (3.1)). We solve this system with respect to $v_{12}$ and $v_{13}$ and obtain that:

$$
v_{12}=-\lambda^{2}, \quad v_{13}=-\lambda^{3}+2 \lambda^{2}-2 \lambda+1 / 2,
$$

and stability polynomial of this family is

$$
p(w, z)=w^{3}(\lambda z-1)^{2}+\frac{1}{2} w^{2}\left(-2 \lambda^{2} z+8 \lambda z-3 z-2\right)+\frac{1}{2} w\left(2 \lambda^{2} z-4 \lambda z+z\right) .
$$

Now we describe search for $A$-stable methods (compare [18], pages 110-112, also pages 258-259). Define

$$
\begin{aligned}
& q(w, y)=p(w, i y), \quad \widehat{q}(w, y)=w^{2} q(1 / w, y) \\
& q_{1}(w, y)=\frac{1}{w}(\widehat{q}(0, y) q(w, y)-q(0, y) \widehat{q}(w, y)) \\
& \widehat{q}_{1}(w, y)=w q_{1}(1 / w, y), \quad q_{0}(y)=\frac{1}{w}\left(\widehat{q}_{1}(0, y) q_{1}(w, y)-q_{1}(0, y) \widehat{q}_{1}(w, y)\right) .
\end{aligned}
$$

Then it follows from Schur theorem ([21]) that all roots of $q(w, y)$ are in the unit circle for all $y \in \mathbb{R}$ if and only if $q_{0}(y)>0$ and $|\widehat{q}(0, y)|>|q(0, y)|$. This leads to system of inequalities:

$$
\left\{\begin{array}{l}
-1 / 2+2 \lambda-4 \lambda^{2}+3 \lambda^{3}>0 \\
-11 / 4 \lambda^{4}+16 \lambda^{5}-25 \lambda^{6}+16 \lambda^{7}-3 \lambda^{8}>0 \\
3 / 4+2 \lambda-3 \lambda^{2}+4 \lambda^{3}>0
\end{array}\right.
$$

which is satisfied for $\lambda \in(0.287159,3.24488)$.

An example of such method corresponding to $\lambda=1$ is

$$
\left[\begin{array}{c|c}
\mathbf{A} & \mathbf{U} \\
\hline \mathbf{B} & \mathbf{V}
\end{array}\right]=\left[\begin{array}{rr|rrr}
1 & 0 & 1 & -1 & 0 \\
1 & 1 & 1 & -1 & -\frac{1}{2} \\
\hline 1 & 1 & 1 & -1 & -\frac{1}{2} \\
-\frac{1}{2} & \frac{3}{2} & 0 & 0 & -\frac{1}{2} \\
-1 & 1 & 0 & 0 & 0
\end{array}\right]
$$

\section{Construction of Methods with $s=3$}

We use representation formulas and obtain a 13-parameter family depending on coefficients of $\mathbf{A}, \mathbf{V}$ and $\mathbf{c}$. Then we set $\mathbf{c}=[1 / 3,2 / 3,1]^{T}$, solve IQS conditions 
(3.2) and (3.3) with respect to $\mathbf{X}, a_{21}, a_{31}, a_{32}, v_{23}, v_{24}$ and $v_{34}$. We obtain

$$
\begin{aligned}
& \mathbf{A}=\left[\begin{array}{ccc}
\lambda & 0 & 0 \\
\frac{1}{3} & \lambda & 0 \\
\frac{1}{3} & \frac{1}{3} & \lambda
\end{array}\right], \quad \mathbf{U}=\left[\begin{array}{cccc}
1 & \frac{1}{3}(1-3 \lambda) & \frac{1}{18}(1-6 \lambda) & \frac{1}{162}(1-9 \lambda) \\
1 & \frac{1}{3}(1-3 \lambda) & \frac{1}{9}(1-6 \lambda) & \frac{1}{162}(5-36 \lambda) \\
1 & \frac{1}{3}(1-3 \lambda) & \frac{1}{6}(1-6 \lambda) & \frac{1}{54}(4-27 \lambda)
\end{array}\right], \\
& \mathbf{B}^{T}=\left[\begin{array}{cccc}
-3 v_{12}+\frac{15}{2} v_{13}-9 v_{14}+\frac{3}{4} & 9 \lambda^{2}-12 \lambda+\frac{11}{6} & 9 \lambda & 9 \\
3 v_{12}-12 v_{13}+18 v_{14} & -18 \lambda^{2}+18 \lambda-\frac{8}{3} & -18 \lambda-3 & -18 \\
v_{12}+\frac{9}{2} v_{13}-9 v_{14}+\frac{1}{4} & 9 \lambda^{2}-6 \lambda+\frac{11}{6} & 9 \lambda+3 & 9
\end{array}\right] \text {, } \\
& \mathbf{V}=\left[\begin{array}{cccc}
1 & v_{12} & v_{13} & v_{14} \\
0 & 0 & \frac{1}{3}(1-6 \lambda) & \frac{1}{27}\left(-27 \lambda^{2}-9 \lambda+2\right) \\
0 & 0 & 0 & \frac{1}{6}(1-6 \lambda) \\
0 & 0 & 0 & 0
\end{array}\right]
\end{aligned}
$$

The stability function of this family of methods is of the form

$p(w, z)=(1-\lambda z)^{3} w^{4}-\left(1+p_{31} z+p_{32} z^{2}+p_{33} z^{3}\right) w^{3}+\left(p_{21} z+p_{22} z^{2}+p_{23} z^{3}\right) w^{2}$, where coefficients $p_{33}$ and $p_{23}$ are given by

$$
\begin{aligned}
& p_{33}=-2 \lambda^{3}-\lambda^{2} v_{12}+3 \lambda^{2}-\frac{\lambda v_{12}}{3}+\lambda v_{13}-\lambda-\frac{v_{12}}{9}+\frac{v_{13}}{2}-v_{14}+\frac{13}{108}, \\
& p_{23}=-\lambda^{3}-\lambda^{2} v_{12}+3 \lambda^{2}+\frac{5 \lambda v_{12}}{3}+\lambda v_{13}-2 \lambda-\frac{5 v_{12}}{18}-\frac{v_{13}}{2}-v_{14}+\frac{1}{4} .
\end{aligned}
$$

We solve system

$$
\left\{\begin{array}{l}
p_{33}=0 \\
p_{23}=0
\end{array}\right.
$$

with respect to $v_{13}, v_{14}$ and obtain a two-parameter family of $L$-stable Nordsieck methods depending on $v_{12}$ and $\lambda$. Remaining parameters can be used to ensure $A$-stability. Use of Schur theorem leads us to a system of six polynomial inequalities, which are not listed here. $\lambda$ and $v_{12}$ for which this inequalities are satisfied are presented in Figure $1 a$.

An example of such method corresponding to $\lambda=1$ and $v_{12}=-2$ is

$$
\left[\begin{array}{c|c}
\mathbf{A} & \mathbf{U} \\
\hline \mathbf{B} & \mathbf{V}
\end{array}\right]=\left[\begin{array}{rrr|rrrr}
1 & 0 & 0 & 1 & -\frac{2}{3} & -\frac{5}{18} & -\frac{4}{81} \\
\frac{1}{3} & 1 & 0 & 1 & -\frac{2}{3} & -\frac{5}{9} & -\frac{31}{162} \\
\frac{1}{3} & \frac{1}{3} & 1 & 1 & -\frac{2}{3} & -\frac{5}{6} & -\frac{23}{54} \\
\hline \frac{8}{9} & -\frac{44}{9} & 7 & 1 & -2 & -\frac{191}{54} & -\frac{62}{27} \\
-\frac{7}{6} & -\frac{8}{3} & \frac{29}{6} & 0 & 0 & -\frac{5}{3} & -\frac{34}{27} \\
9 & -21 & 12 & 0 & 0 & 0 & -\frac{5}{6} \\
9 & -18 & 9 & 0 & 0 & 0 & 0
\end{array}\right] .
$$




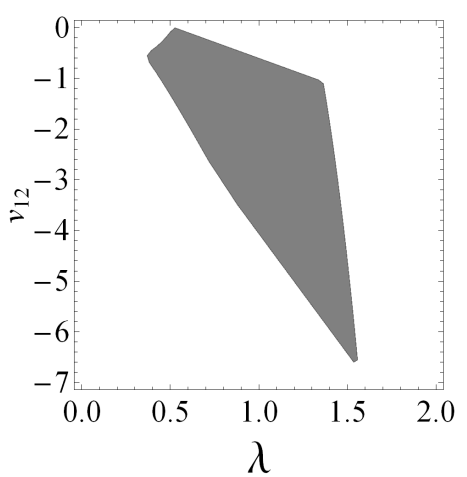

a)

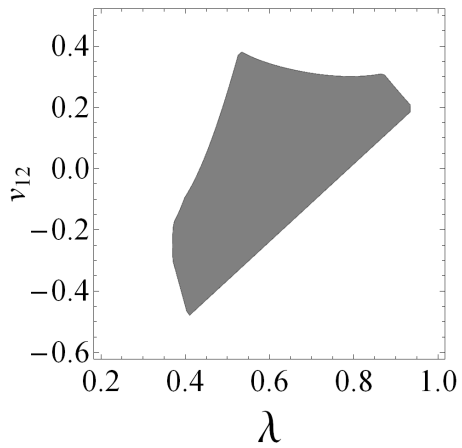

b)

Figure 1. $L$-stable and $A$-stable methods with a) $\mathbf{c}=[1 / 3,2 / 3,1]^{T}$, b) $\mathbf{c}=[0,1,2]^{T}$

If we choose $\mathbf{c}=[0,1,2]^{T}$ we obtain:

$$
\begin{aligned}
& \mathbf{A}=\left[\begin{array}{ccc}
\lambda & 0 & 0 \\
1 & \lambda & 0 \\
1 & 1 & \lambda
\end{array}\right], \mathbf{U}=\left[\begin{array}{cccc}
1 & -\lambda & 0 & 0 \\
1 & -\lambda & \frac{1}{2}(1-2 \lambda) & \frac{1}{6}(1-3 \lambda) \\
1 & -\lambda & 1-2 \lambda & \frac{1}{6}(5-12 \lambda)
\end{array}\right], \\
& \mathbf{B}^{T}=\left[\begin{array}{cccc}
-v_{12}+\frac{3}{2} v_{13}-v_{14}+\frac{5}{12} & \lambda^{2}-3 \lambda+\frac{4}{3} & \lambda-1 & 1 \\
-2 v_{13}+2 v_{14}+\frac{2}{3} & -2 \lambda^{2}+4 \lambda-\frac{2}{3} & 1-2 \lambda & -2 \\
\frac{1}{2} v_{13}-v_{14}-\frac{1}{12} & \lambda^{2}-\lambda+\frac{1}{3} & \lambda & 1
\end{array}\right], \\
& \mathbf{V}=\left[\begin{array}{cccc}
1 & v_{12} & v_{13} & v_{14} \\
0 & 0 & 1-2 \lambda & \frac{1}{6}\left(1-6 \lambda^{2}\right) \\
0 & 0 & 0 & \frac{1}{2}(1-2 \lambda) \\
0 & 0 & 0 & 0
\end{array}\right] .
\end{aligned}
$$

We solve $L$-stability system $p_{33}=0, p_{23}=0$. Parameters $\lambda$ and $v_{12}$ for which methods are also $A$-stable are shown in Figure. $1 b$. An example of such method corresponding to $\lambda=1 / 2$ and $v_{12}=0$ is

$$
\left[\begin{array}{c|c}
\mathbf{A} & \mathbf{U} \\
\hline \mathbf{B} & \mathbf{V}
\end{array}\right]=\left[\begin{array}{rrr|rrrr}
\frac{1}{2} & 0 & 0 & 1 & -\frac{1}{2} & 0 & 0 \\
1 & \frac{1}{2} & 0 & 1 & -\frac{1}{2} & 0 & -\frac{1}{12} \\
1 & 1 & \frac{1}{2} & 1 & -\frac{1}{2} & 0 & -\frac{1}{6} \\
\hline \frac{9}{16} & \frac{1}{2} & -\frac{1}{16} & 1 & 0 & \frac{1}{8} & \frac{1}{24} \\
\frac{1}{12} & \frac{5}{6} & \frac{1}{12} & 0 & 0 & 0 & -\frac{1}{12} \\
-\frac{1}{2} & 0 & \frac{1}{2} & 0 & 0 & 0 & 0 \\
1 & -2 & 1 & 0 & 0 & 0 & 0
\end{array}\right] .
$$




\section{Construction of methods with $s \geq 4$}

Again, first we use representation formulas for $\mathbf{U}$ and $\mathbf{B}$. Then we set $\mathbf{c}=[1 / 4,1 / 2,3 / 4,1]^{T}$. Next we solve IQS conditions with respect to coefficients of matrix $\mathbf{X}$ and $a_{21}, a_{31}, a_{32}, a_{41}, a_{42}, a_{43}, v_{23}, v_{24}, v_{25}, v_{34}, v_{35}$ and $v_{45}$. We obtain a family of Nordsieck methods given by

$$
\begin{aligned}
\mathbf{A} & =\left[\begin{array}{cccc}
\lambda & 0 & 0 & 0 \\
\frac{1}{4} & \lambda & 0 & 0 \\
\frac{1}{4} & \frac{1}{4} & \lambda & 0 \\
\frac{1}{4} & \frac{1}{4} & \frac{1}{4} & \lambda
\end{array}\right], \\
\mathbf{V} & =\left[\begin{array}{ccccc}
1 & v_{12} & v_{13} & v_{14} & v_{15} \\
0 & 0 & \frac{3}{8}-3 \lambda & \frac{7}{48}-\frac{7}{8} \lambda-3 \lambda^{2} & -\frac{1}{64}+\frac{67}{192} \lambda-\frac{15}{8} \lambda^{2}-\lambda^{3} \\
0 & 0 & 0 & \frac{1}{4}-2 \lambda & \frac{11}{192}-\frac{3}{8} \lambda-\lambda^{2} \\
0 & 0 & 0 & 0 & \frac{1}{8}-\lambda \\
0 & 0 & 0 & 0 & 0
\end{array}\right] .
\end{aligned}
$$

Matrices B and $\mathbf{U}$ can be obtained from (2.3),(2.7), and (2.8) and are not listed here.

Range of $v_{12}$ versus $v_{13}$ for some arbitrary chosen $\lambda$ for which methods with coefficients given by (6.1) and (6.2) are $L$ - and $A$ - stable are shown in Figure 2 .

For $\lambda=1, v_{12}=-2, v_{13}=-5$ we obtain an example of such a method:

$$
\begin{aligned}
& \mathbf{A}=\left[\begin{array}{cccc}
1 & 0 & 0 & 0 \\
\frac{1}{4} & 1 & 0 & 0 \\
\frac{1}{4} & \frac{1}{4} & 1 & 0 \\
\frac{1}{4} & \frac{1}{4} & \frac{1}{4} & 1
\end{array}\right], \mathbf{U}=\left[\begin{array}{ccccc}
1 & -\frac{3}{4} & -\frac{7}{32} & -\frac{11}{384} & -\frac{5}{2048} \\
1 & -\frac{3}{4} & -\frac{7}{16} & -\frac{43}{384} & -\frac{29}{1536} \\
1 & -\frac{3}{4} & -\frac{21}{32} & -\frac{1}{4} & -\frac{129}{2048} \\
1 & -\frac{3}{4} & -\frac{7}{8} & -\frac{85}{192} & -\frac{19}{128}
\end{array}\right], \\
& \mathbf{B}=\left[\begin{array}{ccccc}
-\frac{537}{16} & \frac{2971}{24} & -\frac{7531}{48} & \frac{209}{3} \\
-\frac{175}{6} & \frac{1325}{12} & -\frac{863}{6} & \frac{763}{12} \\
-\frac{5}{3} & 35 & -69 & \frac{107}{3} \\
-72 & 232 & -248 & 88 \\
-64 & 192 & -192 & 64
\end{array}\right], \\
& \mathbf{V}=\left[\begin{array}{ccccc}
1 & -2 & -5 & -\frac{1271}{256} & -\frac{1551}{512} \\
0 & 0 & -\frac{21}{8} & -\frac{179}{48} & -\frac{61}{24} \\
0 & 0 & 0 & -\frac{7}{4} & -\frac{253}{192} \\
0 & 0 & 0 & 0 & -\frac{7}{8} \\
0 & 0 & 0 & 0 & 0
\end{array}\right] .
\end{aligned}
$$

Problem of searching highly stable methods with quadratic stability function becomes very difficult for $s>4$. It is still possible to solve IQS conditions 

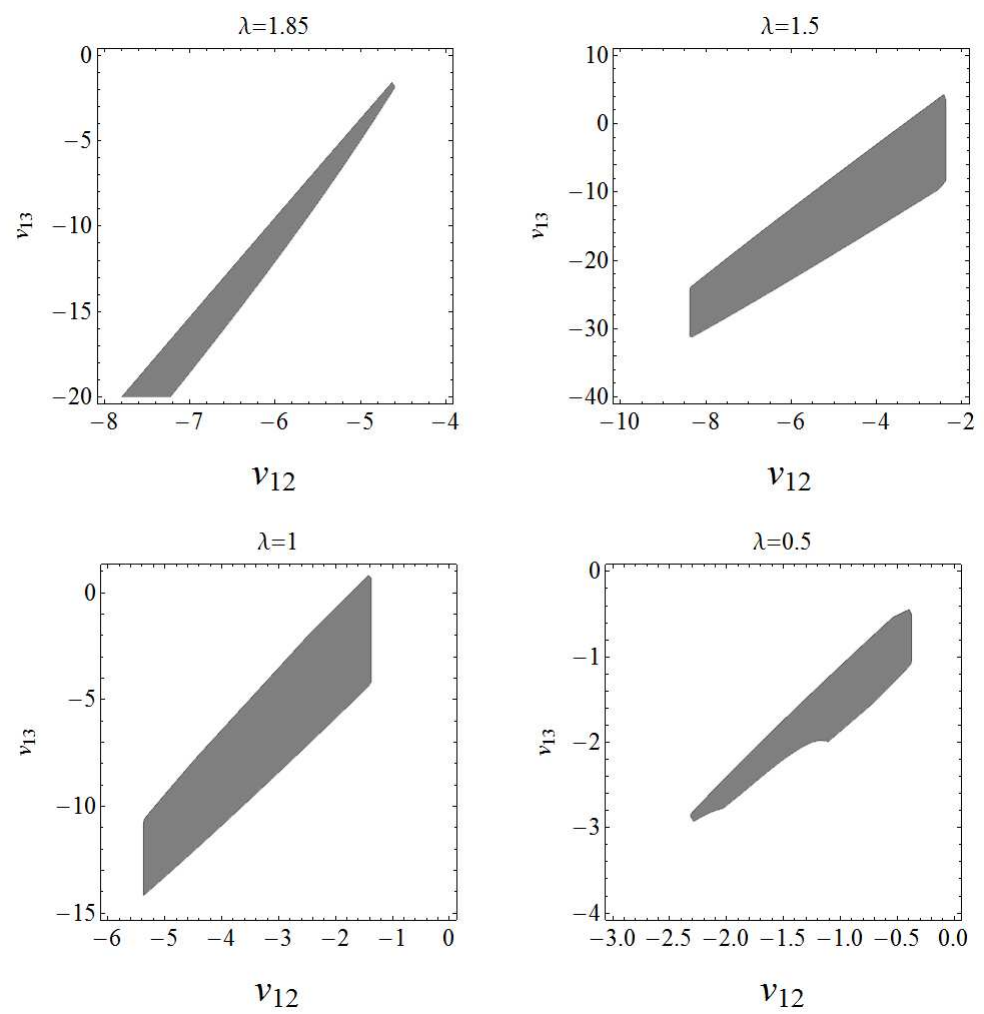

Figure 2. $L$-stable and $A$-stable methods with $\mathbf{c}=[1 / 4,1 / 2,3 / 4,1]^{T}$

for $s=5$. We obtain a six-parameters family of methods of order $p=5$ and stage order $q=5$ depending on $\lambda, v_{12}, v_{13}, v_{14}, v_{15}$ and $v_{16}$ for which the stability function assumes form (3.4). Search for $A$ - and $L$ - stable methods was unsuccessful due to limitations of symbolical manipulation package MATHEMATICA. For $s=6$ we can solve order, stage order and IQS conditions, but we were not able to generate stability function (1.3). We plan to search for such methods in a numerical computing environment such as Matlab.

\section{Numerical Experiments}

In this section we present the results of numerical experiments on the problem of Van der Pol equation (compare [14]):

$$
\left\{\begin{array}{l}
y_{1}^{\prime}=y 2, \quad y_{1}(0)=2 \\
y_{2}^{\prime}=\left(\left(1-y_{1}^{2}\right) y_{2}-y_{1}\right) / \varepsilon, \quad y_{2}(0)=-2 / 3
\end{array}\right.
$$

with integration interval $[0, T], T=3 / 4$ and stiffness parameter $\varepsilon$. 
We rewrite method (1.2) in vector form $([17,18])$ :

$$
\left[\begin{array}{c}
Y^{[n]} \\
\hline z^{[n]}
\end{array}\right]=\left[\begin{array}{c|c}
\mathbf{A} \otimes \mathbf{I} & \mathbf{U} \otimes \mathbf{I} \\
\hline \mathbf{B} \otimes \mathbf{I} & \mathbf{V} \otimes \mathbf{I}
\end{array}\right]\left[\begin{array}{c}
h F\left(Y^{[n]}\right) \\
\hline z^{[n-1]}
\end{array}\right],
$$

where

$$
Y^{[n]}=\left[\begin{array}{c}
Y_{1}^{[n]} \\
\vdots \\
Y_{s}^{[n]}
\end{array}\right], \quad F\left(Y^{[n]}\right)=\left[\begin{array}{c}
f\left(Y_{1}^{[n]}\right) \\
\vdots \\
f\left(Y_{s}^{[n]}\right)
\end{array}\right], \quad z^{[n]}=\left[\begin{array}{c}
z_{1}^{[n]} \\
\vdots \\
z_{s+1}^{[n]}
\end{array}\right]
$$

I is the identity matrix of dimension of the ordinary differential system (1.1) and $\otimes$ is the Kronecker product of two matrices. The vector $Y^{[n]}$ from nonlinear system of equations arising in (7.2) is computed by Newton iteration with initial guess $Y^{0}$ defined as (compare [17]):

$$
Y_{i}^{0}=\sum_{k=1}^{s+1}\left(\frac{c_{i}^{k-1}}{(k-1) !}\right) z_{k}^{[n-1]}, \quad i=1,2, \ldots, s .
$$

We apply method of order $p=q=s=4$ obtained in Section 6 to problem (7.1) with fixed stepsize $h=T / N$ where $N=16,32,64,128,256,512$ and 1024 . In Table 1 we present results of numerical experiments. Here, $\left\|e_{h}(T)\right\|$ is the norm of error at the endpoint of integration and $p$ is the order of convergence given by

$$
p=\frac{\log \left(\left\|e_{h}(T)\right\| /\left\|e_{h / 2}(T)\right\|\right)}{\log (2)} .
$$

Table 1. Numerical results for method of order $p=q=4$.

\begin{tabular}{ccccccc}
\hline & \multicolumn{2}{c}{$\varepsilon=10^{-4}$} & \multicolumn{2}{c}{$\varepsilon=10^{-6}$} & \multicolumn{2}{c}{$\varepsilon=10^{-8}$} \\
$N$ & $\left\|e_{h}(T)\right\|$ & $p$ & $\left\|e_{h}(T)\right\|$ & $p$ & $\left\|e_{h}(T)\right\|$ & $p$ \\
\hline 16 & $5.78 \cdot 10^{-1}$ & & $5.92 \cdot 10^{-1}$ & & $5.92 \cdot 10^{-1}$ & \\
32 & $6.92 \cdot 10^{-2}$ & 3.06 & $7.22 \cdot 10^{-2}$ & 3.04 & $7.22 \cdot 10^{-2}$ & 3.04 \\
64 & $4.01 \cdot 10^{-3}$ & 4.11 & $4.33 \cdot 10^{-3}$ & 4.06 & $4.33 \cdot 10^{-3}$ & 4.06 \\
128 & $1.17 \cdot 10^{-4}$ & 5.10 & $1.39 \cdot 10^{-4}$ & 4.96 & $1.39 \cdot 10^{-4}$ & 4.96 \\
256 & $1.03 \cdot 10^{-6}$ & 6.82 & $2.48 \cdot 10^{-6}$ & 5.81 & $2.58 \cdot 10^{-6}$ & 5.75 \\
512 & $6.82 \cdot 10^{-8}$ & 3.92 & $3.51 \cdot 10^{-8}$ & 6.14 & $8.28 \cdot 10^{-8}$ & 4.96 \\
1024 & $6.51 \cdot 10^{-9}$ & 3.39 & $7.28 \cdot 10^{-10}$ & 5.59 & $2.09 \cdot 10^{-9}$ & 5.31 \\
\hline
\end{tabular}

These results illustrate that the method achieves the expected order of accuracy for stiff differential system and do not suffer from order reduction phenomenon. 


\section{Concluding Remarks}

In this paper a new criteria for quadratic stability was given. Based on this results and the Schur theorem new families of $L$ - and $A$-stable methods up to order 4 were constructed. Further work will be concerned with the implementation aspects including a choice of initial stepsize, local error estimation for small and large stepsizes, step size and order changing strategies, construction of continuous extensions, approximation of the Jacobian matrix, and solving nonlinear equations by simplified Newton iterations at each step of the integration.

\section{Acknowledgments}

The author would like to express his gratitude to Professor Zdzisław Jackiewicz for many useful discussions and for helping prepare this article and to Raffaele D'Ambrosio for assistance in numerical experiment. Further, the author wishes to thank anonymous referee for his constructive comments.

\section{References}

[1] Z. Bartoszewski and Z. Jackiewicz. Nordsieck representation of two-step Runge-Kutta methods for ordinary differential equations. Appl. Numer. Math, 53(2):149-163, 2005. Doi:10.1016/j.apnum.2004.08.010.

[2] J.C. Butcher. The equivalence of algebraic stability and AN-stability. BIT, 27(4):510-533, 1987. Doi:10.1007/BF01937275.

[3] J.C. Butcher. The Numerical Analysis of Ordinary Differential Equations. Runge-Kutta and General Linear Methods. John Wiley \& Sons, Chichester, New York, 1987.

[4] J.C. Butcher. Numerical Methods for Ordinary Differential Equations. John Wiley \& Sons, Chichester, 2003. Doi:10.1002/9780470753767.

[5] J.C. Butcher, P. Chartier and Z. Jackiewicz. Nordsieck representation of DIMSIMs. Numer. Algorithms, 16(2):209-230, 1997. Doi:10.1023/A:1019195215402.

[6] J.C. Butcher, P. Chartier and Z. Jackiewicz. Experiments with a variableorder type 1 DIMSIM code. Numer. Algorithms, 22(3-4):237-261, 1999. Doi:10.1023/A:1019135630307.

[7] J.C. Butcher and Z. Jackiewicz. Implementation of diagonally implicit multistage integration methods for ordinary differential equations. SIAM J. Numer. Anal., 34(6):2119-2141, 1997. Doi:10.1137/S0036142995282509.

[8] J.C. Butcher and Z. Jackiewicz. Error estimation for Nordsieck methods. Numer. Algorithms, 31(1-4):75-85, 2002. Doi:10.1023/A:1021152005287.

[9] J.C. Butcher and H. Podhaisky. On error estimation in general linear methods for stiff ODEs. Appl. Numer. Math., 56(3-4):345-357, 2006. Doi:10.1016/j.apnum.2005.04.013.

[10] J.C. Butcher and W.M. Wright. The construction of practical general linear methods. BIT, 43(4):695-721, 2003. Doi:10.1023/B:BITN.0000009952.71388.23. 
[11] R. Čiegis, O. Iliev, V. Starikovičius and K. Steiner. Numerical algorithms for solving problems of multiphase flows in porous media. Mathematical Modelling and Analysis, 11(2):133-148, 2006.

[12] D. Conte, R. D'Ambrosio and Z. Jackiewicz. Two-step Runge-Kutta methods with quadratic stability functions. J. Sci. Comput., 44(2):191-218, 2010. Doi:10.1007/s10915-010-9378-x.

[13] R. D'Ambrosio, G. Izzo and Z. Jackiewicz. Search for highly stable two-step Runge-Kutta methods. Appl. Numer. Math. (accepted)

[14] E. Hairer and G. Wanner. Solving Ordinary Differential Equations II. Stiff and Differential-Algebraic Problems, Second Revised Edition. Springer Verlag, Berlin, Heidelberg, New Yor, 1996.

[15] L.L. Hewitt and A.T. Hill. Algebraically stable general linear methods and the G-matrix. BIT, 49(1):93-111, 2009. Doi:10.1007/s10543-008-0207-7.

[16] A.T. Hill. Nonlinear stability of general linear methods. Numer. Math., 103(4):611-629, 2006. Doi:10.1007/s00211-006-0011-8.

[17] Z. Jackiewicz. Implementation of DIMSIMs for stiff differential systems. Appl. Numer. Math., 42(1):251-267, 2002. Doi:10.1016/S0168-9274(01)00154-4.

[18] Z. Jackiewicz. General Linear Methods for Ordinary Differential Equations. John Wiley \& Sons, Hoboken, New Jersey, 2009. Doi:10.1002/9780470522165.

[19] Z. Jackiewicz, Y. Kuang, C. Thalhauser and B. Zubik-Kowal. Numerical solution of a model for brain cancer progression after therapy. Mathematical Modelling and Analysis, 14(1):43-56, 2009. Doi:10.3846/1392-6292.2009.14.43-56.

[20] Z. Jackiewicz and S. Tracogna. A general class of two-step Runge-Kutta methods for ordinary differential equations. SIAM J. Numer. Anal., 32(5):1390-1427, 1995. Doi:10.1137/0732064.

[21] J. Schur. über potenzreihen die im innern des einheitskreises beschränkt sind. J. Reine Angew. Math., 147:205-232, 1917. Doi:10.1515/crll.1917.147.205.

[22] W. Wright. General linear methods with inherent Runge-Kutta stability, Ph.D. thesis. University of Auckland, New Zealand, 2002. 